\title{
Novel electrophilic aromatic iodine substitution of some naphtholic Schiff bases
}

\author{
Murat Gündüz, Sevim Bilgiç," Orhan Bilgiç, and Devrim Özöğüt \\ Department of Chemistry, Faculty of Arts and Science, University of \\ Eskişehir Osmangazi, 26480, Eskişehir, Turkey
}

E-mail: sbilgic@ogu.edu.tr

\begin{abstract}
During the ring closure trial of the naphtholic Schiff bases (1a-d) we found that iodine was incorporated into the phenyl ring. The structures of the products (2a-e) obtained with this novel substitution reaction, were determined by NMR $\left({ }^{1} \mathrm{H}-\mathrm{NMR},{ }^{13} \mathrm{C}-\mathrm{NMR}, 2 \mathrm{D}-\mathrm{NMR}\right)$, mass, IR and UV spectra as well as chemical analysis.
\end{abstract}

Keywords: Aromatic iodination, electrophilic aromatic substitution, iodoarenes, Schiff base

\section{Introduction}

Iodoaromatic compounds are valuable and versatile synthetic intermediates in many domains of synthetic organic chemistry, medicine and biochemistry. ${ }^{1,2}$ Direct iodination of aromatic compounds is difficult due to the poor electrophilic strength of molecular iodine compared to that of molecular chlorine and bromine. Iodination of aromatic compounds was carried out by using molecular iodine together with Lewis acid or a strong oxidizing agent such as $\mathrm{Ag}_{2} \mathrm{SO}_{4}$, $\mathrm{NaIO}_{4}, \mathrm{HIO}_{4}, \mathrm{NaOCl}$, liquid $\mathrm{SO}_{3}$, iodic acid, ${ }^{3}$ nitric acid and sulfuric acid, ${ }^{4}$ sulfur trioxide and hydrogen peroxide. ${ }^{5-9}$ Recently, direct iodination methods have been reported using various iodinium donating reagents, such as iodine $/ \mathrm{Na}_{2} \mathrm{~S}_{2} \mathrm{O}_{8}{ }^{10}$ iodine- $\left(\mathrm{NH}_{4}\right)_{2} \mathrm{~S}_{2} \mathrm{O}_{8}-\mathrm{CuCl}_{2}-\mathrm{Ag}_{2} \mathrm{SO}_{4}{ }^{11}$ $\mathrm{NaOCl}-\mathrm{NaI},{ }^{12}$ bis(pyridineiodonium(I)tetrafluoroborate- $\mathrm{CF}_{3} \mathrm{SO}_{3} \mathrm{H},{ }^{13}$ iodine silver sulfate, ${ }^{14} \mathrm{NIS}$ $\mathrm{CF}_{3} \mathrm{SO}_{3} \mathrm{H}^{2}$ iodine-mercury salts, ${ }^{15}$ iodine monochloride, ${ }^{16}$ mercury(II)-oxide-iodine, ${ }^{17}$ bis(symcollidine)iodine(I)hexafluorophosphate, ${ }^{18}$ iodine-di-iodine pentoxide, $^{19}$ NIS ( $N$-iodosuccinimide) ${ }^{20}$ iodine-nitrogendioxide, ${ }^{21}$ iodine-F-TEDA-BF $4,{ }^{22}$ iodine-iodic acid, ${ }^{23} n$-BuLi$\mathrm{CF}_{3} \mathrm{CH}_{2} \mathrm{I},{ }^{24} \mathrm{NH}_{4} \mathrm{I}$-oxone, ${ }^{25} \mathrm{ICl}^{26}$

However, most of these methods require a high reaction temperature and long reaction times, ${ }^{20}$ work in low yields, ${ }^{27}$ involve toxic heavy metals, involve harsh conditions, and generate costly or complex and hazardous waste..$^{25,28-30}$ Therefore, attempts have been made to overcome 
these disadvantages, by employing recyclable heterogeneous catalysts with a wide variety of reagents and conditions. In this paper, we report an exceedingly simple, inexpensive, quick, new, mild, selective and environmentally attractive method for direct aromatic iodination, using the DMSO- $\mathrm{H}^{+}-\mathrm{I}_{2}$ reagent system. The reaction conditions were discovered when exploring the ring closure of some naphtholic Schiff bases (1a-d), according to the method of Lokhande and Ghiya, ${ }^{31}$ yielding iodo substituted Schiff bases (2a-e).

\section{Results and Discussion}

Naphtholic Schiff bases (1a-d) were synthesized according to the method of Sawich and coworkers. ${ }^{32}$ Mass spectra revealed that electrophilic substitution instead of aimed ring closure had occurred (3). There were two possibilities for the iodine substitution: (a) substitution of iodine in position 3 of the naphthalene ring yielding compound (4) (b) substitution of iodine in the phenyl ring yielding the compounds (2a-e).
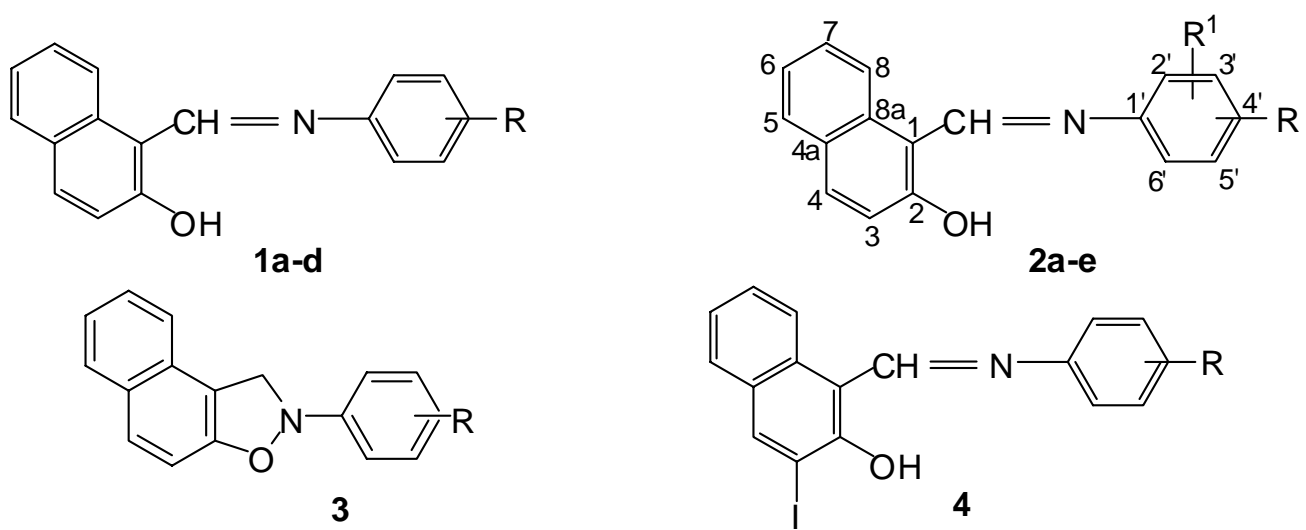

$$
\begin{aligned}
& \text { 1a. } \mathrm{R}=\mathrm{H} \\
& \text { 1b. } \mathrm{R}=2^{\prime}-\mathrm{CH}_{3} \\
& \text { 1c. } \mathrm{R}=3^{\prime}-\mathrm{CH}_{3} \\
& \text { 1d. } \mathrm{R}=4^{\prime}-\mathrm{CH}_{3} \\
& \hline
\end{aligned}
$$

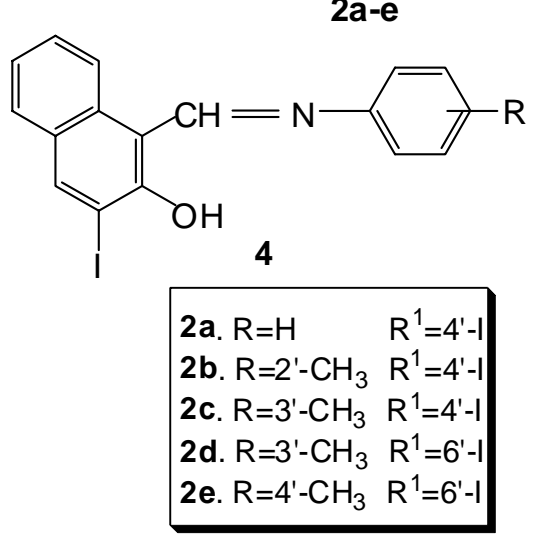

\section{Scheme 1}

The structure of the Schiff base (1a-e) substrates prepared was determined by IR, UV, ${ }^{1} \mathrm{H}-$ NMR and ${ }^{13} \mathrm{C}$-NMR spectra and compared with literature data. The naphtholic Schiff base 1a was reacted with $\mathrm{DMSO} / \mathrm{H}^{+}-\mathrm{I}_{2}$ for 12 hours. Preparative TLC $\left(\mathrm{SiO}_{2} /\right.$ toluene) of the crude product (2a) showed the presence of one new compound $\left(\mathrm{R}_{\mathrm{f}}=0.24\right)$ together with the unreacted Schiff base (1a). The new product was crystallized from alcohol yielding yellow crystals ( $\mathrm{mp}=142-144$ $\left.{ }^{\circ} \mathrm{C}\right)$. Mass spectrum of the compound showed a molecular ion peak at 373.0 and a $(\mathrm{M}-\mathrm{HI})^{+}$peak at 245.0. In the IR spectrum there were $(-\mathrm{CH}=\mathrm{N}-)$ and $-\mathrm{OH}$ groups at $1625 \mathrm{~cm}^{-1}$ and $3500 \mathrm{~cm}^{-1}$. 
${ }^{13} \mathrm{C}-\mathrm{NMR}$ spectrum $\left(\mathrm{CDCl}_{3}\right)$ of the product showed a peak at $90.8 \mathrm{ppm}$ which can be attributed to the iodine substituted carbon. So instead of the ring closure, iodine was substituted into the phenyl ring of the Schiff base (2a). In the ${ }^{1} \mathrm{H}-\mathrm{NMR}$ spectrum $\left(\mathrm{CDCl}_{3}\right)$ of the product one-proton singlet at $15.25 \mathrm{ppm}$ was attributed to the naphtholic $-\mathrm{OH}$ and two doublets at 7.11 and $7.76 \mathrm{ppm}$ revealed that the iodine was substituted in para-position to the azomethine group $(-\mathrm{CH}=\mathrm{N}-)$ in the phenyl ring. From the HETCOR $\left(\mathrm{CDCl}_{3}\right)$ of the compound we assigned the signal to the azomethine group at 9.33 (155.6), and the signals 8.09 (136.7), 7.82 (129.4), 7.76 (138.7), 7.73 (121.6), 7.11 (122.4), 7.11 (118.9) were assigned to the H-4 (C-4), H-5 (C-5), H-3' and H-5' (C$3^{\prime}$ and $\left.\mathrm{C}-5^{\prime}\right), \mathrm{H}-8$ (C-8), H-2' (C-2') and H-3 (C-3) carbons and protons respectively. The other two signals at 7.53 (128.2) and 7.35 (123.7) were due to the H-7 (C-7) and H-6 (C-6) protons and carbons of the product. These spectroscopic data and the chemical analysis where in accordance with 1-\{[(4-iodophenyl)imino]methyl $\}$-2-naphthol (2a).

Since we obtained the iodine substituted Schiff base 2a instead of ring closed product $\mathbf{3 a}$ we tried iodine substitution of Schiff base $\mathbf{1 b}$, bearing an $o$-methyl group, with DMSO- $\mathrm{I}_{2}-\mathrm{H}^{+}$under the same reaction conditions. The crude mixture showed the presence of one new compound together with the unreacted Schiff base 1b. It was purified with preparative TLC $\left(\mathrm{SiO}_{2} /\right.$ toluene) $\left(\mathrm{R}_{\mathrm{f}}=0.26\right)$ and crystallized from alcohol (yellow crystals, mp: $\left.136-138{ }^{\circ} \mathrm{C}\right)$. Mass spectrum of the purified compound $\mathbf{2 b}$ gave a molecular ion peak at 387.0 confirming iodine substitution. Chemical analysis of the compound was in agreement with the iodine substituted structure. In the IR spectrum there were $-\mathrm{OH}$ and $-\mathrm{CH}=\mathrm{N}$ - peaks at 3500 and $1625 \mathrm{~cm}^{-1}$. In ${ }^{13} \mathrm{C}$-NMR spectrum of 2b there were $\mathrm{C}-\mathrm{I}$ and $-\mathrm{CH}=\mathrm{N}$ - peaks at 90.9 and $154.8 \mathrm{ppm}$ respectively and together with the other peaks they confirmed the Schiff base structure $\mathbf{2 b}$. From the ${ }^{1} \mathrm{H}-\mathrm{NMR}$ spectrum of the product it was clear that the substitution occurred to the para-position of the phenyl ring with respect to the azomethine group.

Since in the $o$-methyl substituted Schiff base (1b) iodine was substituted to the para-position of the phenyl ring (2b), we investigated the effect of a $m$-methyl group in the reaction. When applying the same reaction conditions, two compounds (2c and $\mathbf{2 d}$ ) were isolated by preparative TLC $\left(\mathrm{SiO}_{2} /\right.$ toluene). The compound with $\mathrm{R}_{\mathrm{f}}=0.25$ was crystallized from alcohol delivering yellow crystals (mp: $96{ }^{\circ} \mathrm{C}$ ). ${ }^{1} \mathrm{H}-\mathrm{NMR}$ and ${ }^{13} \mathrm{C}-\mathrm{NMR}$ spectra showed that iodine again was substituted in para-position of the phenyl ring with respect to the azomethine group (2c). For the compound with $\mathrm{R}_{\mathrm{f}}=0.46$ (yellow crystals, mp: $146-148{ }^{\circ} \mathrm{C}$ ) mass spectra and the chemical analysis confirmed the iodine substituted Schiff base (2d) with iodine substituted in the orthoposition with respect to the azomethine group and para-position relative to the $-\mathrm{CH}_{3}$ group. ${ }^{1} \mathrm{H}$ $\mathrm{NMR}$ and ${ }^{13} \mathrm{C}$-NMR spectra as well as the mass spectrum and chemical analysis were in agreement with this structure.

Subsequently, with a blocked para-position we wished to explore the effect on the substitution reaction. After heating of the Schiff base 1e with DMSO- $\mathrm{I}_{2}-\mathrm{H}^{+}$under the same reaction condition we obtained one product $(2 \mathrm{e})\left(\mathrm{mp}: 142{ }^{\circ} \mathrm{C}\right)$ together with the unreacted Schiff base (1e). Mass spectrum of the isolated product was again 387.0 and chemical analysis confirmed the expected elemental composition of 2e. Analysis of ${ }^{1} \mathrm{H}-\mathrm{NMR}$ and ${ }^{13} \mathrm{C}-\mathrm{NMR}$ spectra 
showed that iodine was substituted in the ortho-position with respect to the azomethine group and meta-position relative to the $-\mathrm{CH}_{3}$ group in the phenyl ring. For the mechanism of the iodine substitution reaction we suggest the mechanism in Scheme 2.
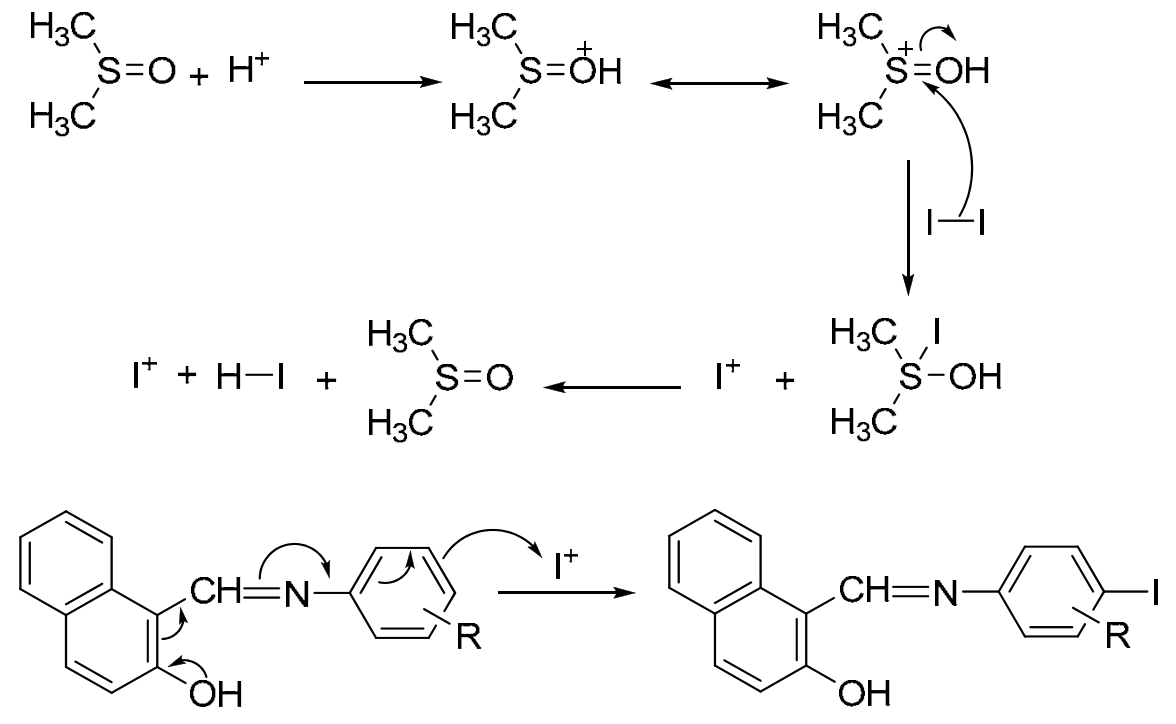

Scheme 2. Electrophilic aromatic iodine substitution mechanism.

\section{Experimental Section}

General Procedures. All melting points were taken in open capillaries and uncorrected. The IR spectra in $\mathrm{KBr}$ were recorded on Mattson 1000 FTR spectrometer and JASCO ST / IR-420 machine and UV spectra on Unicam UV2-100/Visible spectrometer and 150-20 Hitachi spectrometer. ${ }^{1} \mathrm{H}$ and ${ }^{13} \mathrm{C}-\mathrm{NMR}$ spectra were determined at Bruker AC 200L and Bruker 400 $\mathrm{MHz}$ spectrometer using $\mathrm{CDCl}_{3}$. Mass spectra were obtained in a (LS/MS-APCl) Agilent 1100 MSD Instrument. Elemental analyses were obtained LECO CHNS 932 Machine. Merck Kieselgel $\mathrm{HF}_{254}$ type-60 and Kieselgel 40-60 $\mu \mathrm{m}$ type were used for TLC. For analytical work $0.25 \mathrm{~mm}$, for preparative work $0.75 \mathrm{~mm}$ plates were used. All solvents and reagents used were analytical reagent grade.

\section{The synthesis of the Schiff bases (1a-d)}

Schiff bases (1a-d) were synthesized according to the method of Sawich and coworkers. ${ }^{31}$ Their structures were determined by IR, UV and ${ }^{13} \mathrm{C}-\mathrm{NMR}$ spectra.

\section{Reaction of Schiff bases (1a-d) with DMSO-H ${ }^{+}-\mathbf{I}_{2}$}

Schiff bases (1a-d) (4 mmol) were dissolved in DMSO $(16 \mathrm{ml})$ and the $\mathrm{H}_{2} \mathrm{SO}_{4}$ was added until the $\mathrm{pH}$ was approximately 5.0 and then catalytic amount $\mathrm{I}_{2}$ for (1a), for the others (1)-d) 
equivalent amount ( $4 \mathrm{mmol}) \mathrm{I}_{2}$ was added to this solution. The reaction mixture was heated at $125-130{ }^{\circ} \mathrm{C}$ for 12 hours under $\mathrm{N}_{2}$ atmosphere. The crude product was washed with iced water to remove DMSO and dissolved with $10 \% \mathrm{Na}_{2} \mathrm{~S}_{2} \mathrm{O}_{3}$ to remove the excess iodine. Then the crude product was extracted with $\mathrm{CH}_{2} \mathrm{Cl}_{2}(30 \mathrm{ml})$. After evaporation of the solvent in vacuo crude product was purified by preparative $\mathrm{TLC}\left(\mathrm{SiO}_{2} /\right.$ toluene) and crystallized from ethanol.

1-\{[(4-iodophenyl)imino]methyl\}-2-naphthol (2a). Yield: 17.6\%. mp: 142-144 ${ }^{\circ} \mathrm{C}$. Mass spectrum: $\mathrm{m} / \mathrm{z}=[373.0,100 \%], 245.0[\mathrm{M}-\mathrm{HI}, 41.6 \%]^{+}, 227.0\left[\mathrm{M}-\left(\mathrm{HI}+\mathrm{H}_{2} \mathrm{O}\right), 31.6 \%\right]^{+}$. Anal: Calcd for $\mathrm{C}_{17} \mathrm{H}_{12} \mathrm{INO}$ (373.19): C, 54.71; H, 3.24; N, 3.75. Found: $\mathrm{C}, 54.16 ; \mathrm{H}, 4.35 ; \mathrm{N}, 3.23$. IR $(\mathrm{KBr}) v_{\max }: 3500,1625,1574-1497 \mathrm{~cm}^{-1} . \mathrm{UV}(\mathrm{EtOH}) \lambda_{\max }(\log \varepsilon): 284.4(0.412), 317.2(0.585)$, 357.2 (0.414), $461.6 \mathrm{~nm}(0.070) .{ }^{1} \mathrm{H}-\mathrm{NMR}\left(400 \mathrm{MHz}, \mathrm{CDCl}_{3}\right): \delta 7.11(2 \mathrm{H}, \mathrm{d}, J=8.5 \mathrm{~Hz}, \mathrm{H}-3$ and H-2'), $7.35(1 \mathrm{H}, \mathrm{dt}, J=1.02,7.7 \mathrm{~Hz}, \mathrm{H}-6), 7.53(1 \mathrm{H}, \mathrm{dt}, J=1.02,7.4 \mathrm{~Hz}, \mathrm{H}-7), 7.73(1 \mathrm{H}, \mathrm{d}, J=9.0$ $\mathrm{Hz}, \mathrm{H}-8), 7.76\left(2 \mathrm{H}, \mathrm{d}, J=8.5 \mathrm{~Hz}, \mathrm{H}-3^{\prime}\right), 7.81(1 \mathrm{H}, \mathrm{d}, J=9.0 \mathrm{~Hz}, \mathrm{H}-5), 8.09$ (1H, d, $\left.J=8.5 \mathrm{~Hz}, \mathrm{H}-4\right)$, $9.33\left(1 \mathrm{H}, \mathrm{d}, J=1.82 \mathrm{~Hz},(-\mathrm{CH}=\mathrm{N}-), 15.25(1 \mathrm{H}\right.$, brd.s, naphtholic $-\mathrm{OH}) .{ }^{13} \mathrm{C}-\mathrm{NMR}(400 \mathrm{MHz}$, $\left.\mathrm{CDCl}_{3}\right): \delta 90.8\left(\mathrm{C}-4^{\prime}\right), 109.0(\mathrm{C}-1), 118.9(\mathrm{C}-3), 121.6(\mathrm{C}-8), 122.4\left(\mathrm{C}-2^{\prime}\right), 123.7$ (C-6), 127.5 (C-4a), 128.2 (C-7), 129.4 (C-5), 133.0 (C-8a), 136.7 (C-4), 138.7 (C-3'), 145.8 (C-1'), 155.6 ($\mathrm{CH}=\mathrm{N}-), 168.9(\mathrm{C}-2)$. HETCOR $\left(\mathrm{CDCl}_{3}\right)$ : 9.33, $-\mathrm{CH}=\mathrm{N}-(155.6,-\mathrm{CH}=\mathrm{N}-$ azomethine carbon), 8.09, H-4 (136.7, C-4), 7.81, H-5 (129.4, C-5), 7.76, H-3' (138.7, C-3'), 7.73, H-8 (121.6, C-8), 7.53, H-7 (128.2, C-7), 7.35, H-6 (123.7, C-6), 7.11, H-2' (122.4, C-2'), 7.11, H-3 (118.9, C-3).

1-\{[(4-iodo-2-methylphenyl)imino]methyl\}-2-naphthol (2b). Yield: $39.4 \% . \mathrm{mp:} 136-138{ }^{\circ} \mathrm{C}$. Mass spectrum: $\mathrm{m} / \mathrm{z}=\mathrm{M}^{+}$[387.0], [259.0 (M-HI, 23.6\%)] ${ }^{+}$, [244.0 ( $\left.\left(\mathrm{M}_{-} \mathrm{C}_{10} \mathrm{H}_{8} \mathrm{O}, 16.3 \%\right)\right]^{+}$. Anal: Calcd for $\mathrm{C}_{18} \mathrm{H}_{14} \mathrm{INO}$ (387.21): C, 55.83; H, 3.64; N, 3.62. Found: $\mathrm{C}, 56.40 ; \mathrm{H}, 4.55 ; \mathrm{N}, 3.34$. IR (KBr) $v_{\max }: 3500,1625,1600-1497 \mathrm{~cm}^{-1} . \mathrm{UV}(\mathrm{EtOH}) \lambda_{\max }(\log \varepsilon): 288.0(0.701), 322(0.250)$, 360.0 (0.148), $445.2(0.139), 466.0 \mathrm{~nm}(0.135) .{ }^{1} \mathrm{H}-\mathrm{NMR}\left(400 \mathrm{MHz}, \mathrm{CDCl}_{3}\right): \delta 2.41(3 \mathrm{H}, \mathrm{s},-$ $\left.\mathrm{CH}_{3}\right), 7.02\left(1 \mathrm{H}, \mathrm{d}, J \cong 8.5, \mathrm{H}-6^{\prime}\right), 7.09(1 \mathrm{H}, \mathrm{d}, J=9.0 \mathrm{~Hz}, \mathrm{H}-3), 7.34(1 \mathrm{H}, \mathrm{dt}, J \cong 7.5 \mathrm{~Hz}, \mathrm{H}-6), 7.52$ $(1 \mathrm{H}, \mathrm{dt}, J=7.5, \mathrm{H}-7), 7.62\left(1 \mathrm{H}, \mathrm{d}, J \cong 7.0 \mathrm{~Hz}, \mathrm{H}-5^{\prime}\right), 7.62\left(1 \mathrm{H}\right.$, brd.s, $\left.\mathrm{H}-3^{\prime}\right), 7.82(1 \mathrm{H}, \mathrm{d}, J=9.0 \mathrm{~Hz}$, $\mathrm{H}-5), 7.72(1 \mathrm{H}, \mathrm{d}, J=8.0 \mathrm{~Hz}, \mathrm{H}-8), 8.10(1 \mathrm{H}, \mathrm{d}, J \cong 8.5 \mathrm{~Hz}, \mathrm{H}-4), 9.30(1 \mathrm{H}, \mathrm{d}, J=3.0 \mathrm{~Hz},-\mathrm{C} \underline{H}=\mathrm{N})$, $15.51\left(1 \mathrm{H}\right.$, brd.s, naphtholic -OH). ${ }^{13} \mathrm{C}$ NMR $\left(400 \mathrm{MHz}, \mathrm{CDCl}_{3}\right): \delta 17.8\left(-\mathrm{CH}_{3}\right), 90.9\left(\mathrm{C}-4^{\prime}\right)$, 109.7 (C-1), 118.8 (C-6'), 118.9 (C-3), 121.8 (C-8), 123.7 (C-6), 127.4 (C-4a), 128.2 (C-7), 129.4 (C-5), 133.0 (C-8a), 133.6 (C-2'), 136.2 (C-4), 136.8 (C-5'), 139.7 (C-3'), 144.4 (C-1'), $154.8(-\mathrm{CH}=\mathrm{N}-), 169.5(\mathrm{C}-2)$.

1-\{[(4-iodo-3-methylphenyl)imino]methyl\}-2-naphthol (2c). Yield: $29.2 \%$. mp: $96{ }^{\circ} \mathrm{C}$. Mass

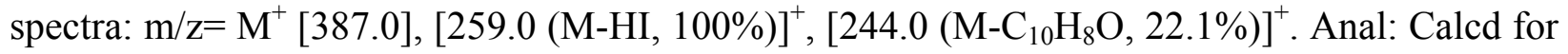
$\mathrm{C}_{18} \mathrm{H}_{14} \mathrm{INO}$ (387.21): C, 55.83; H, 3.64; N, 3.62. Found: C, 56.49; H, 3.76; N, 3.39. IR (KBr) $v_{\max }: 3150,1625,1574-1497 \mathrm{~cm}^{-1}$. UV (EtOH) $\lambda_{\max }(\log \varepsilon): 286.4(0.560), 316.8(0.560), 358.8$ (0.396), $443.6(0.080), 464.0(0.079) \mathrm{nm} .{ }^{1} \mathrm{H}-\mathrm{NMR}\left(400 \mathrm{MHz}, \mathrm{CDCl}_{3}\right): \delta 2.45\left(3 \mathrm{H} \mathrm{s},-\mathrm{CH}_{3}\right), 6.86$ $\left(1 \mathrm{H}, \mathrm{d}, J \cong 7.5 \mathrm{~Hz}, \mathrm{H}-6^{\prime}\right), 7.05(1 \mathrm{H}, \mathrm{d}, J \cong 9.0 \mathrm{~Hz}, \mathrm{H}-3), 7.17\left(1 \mathrm{H}, \mathrm{s}, \mathrm{H}-2^{\prime}\right), 7.31(1 \mathrm{H}, \mathrm{t}, J=7.5 \mathrm{~Hz}$, H-6), 7.49 (1H, t, J=7.8 Hz, H-7), $7.68\left(1 \mathrm{H}, \mathrm{d}, J \cong 8.0 \mathrm{~Hz}, \mathrm{H}-5^{\prime}\right), 7.76(1 \mathrm{H}, \mathrm{d}, J=7.5 \mathrm{~Hz}, \mathrm{H}-8), 7.81$ $(1 \mathrm{H}, \mathrm{d}, J \cong 9.0 \mathrm{~Hz}, \mathrm{H}-5), 8.05(1 \mathrm{H}, \mathrm{d}, J \cong 8.5 \mathrm{~Hz}, \mathrm{H}-4), 9.24(1 \mathrm{H}$, brd.s, $-\mathrm{C} \underline{\mathrm{H}}=\mathrm{N}-), 15.36(1 \mathrm{H}$, brd.s, naphtholic -OH). ${ }^{13} \mathrm{C}$ NMR (400 MHz, $\left.\mathrm{CDCl}_{3}\right): \delta 22.9\left(-\mathrm{CH}_{3}\right), 97.6(\mathrm{C}-4$ '), $108.9(\mathrm{C}-1), 118.9$ (C-2'), 119.1 (C-3), 121.6 (C-8), 121.9 (C-6'), 123.6 (C-6) 127.3 (C-4a), 128.1 (C-7), 129.4 (C- 
5), 133.1 (C-8a), 136.8 (C-4), 139.8 (C-5'), 142.8 (C-3'), 145.5 (C-1'), 154.7 (-CH=N-), 169.8 (C-2).

1-\{[(2-iodo-5-methylphenyl)imino]methyl\}-2-naphthol (2d). Yield: $19.4 \%$. mp: $146-148{ }^{\circ} \mathrm{C}$. Mass spectra: $\mathrm{m} / \mathrm{z}=\mathrm{M}^{+}$[387.0], [259.0 (M-HI, 39.3\%)] ${ }^{+},\left[106.1\left(\mathrm{C}_{8} \mathrm{H}_{10}, 100 \%\right)\right]^{+}$. Anal: Calcd for $\mathrm{C}_{18} \mathrm{H}_{14} \mathrm{INO}$ (387.21): C, 55.83; H, 3.64; N, 3.62. Found: C, 56.02; H, 4.17; N, 2.97. IR (KBr) $v_{\max }: 3500,1625,1574-1497 \mathrm{~cm}^{-1}$. UV (EtOH) $\lambda_{\max }(\log \varepsilon): 288.4(0.738), 316.8(0.376), 338.8$ (0.376), 359.6 (0.419), $462.4(0.090) \mathrm{nm} .{ }^{1} \mathrm{H}-\mathrm{NMR}\left(400 \mathrm{MHz}, \mathrm{CDCl}_{3}\right): \delta 2.39\left(3 \mathrm{H}, \mathrm{s},-\mathrm{CH}_{3}\right)$, $6.81\left(1 \mathrm{H}, \mathrm{d}, J=8.0 \mathrm{~Hz}, \mathrm{H}-4^{\prime}\right), 7.04\left(1 \mathrm{H}, \mathrm{s}, \mathrm{H}-2^{\prime}\right), 7.18\left(1 \mathrm{H}, \mathrm{d}, J \cong 9.0 \mathrm{~Hz}, \mathrm{H}-5^{\prime}\right.$ ve H-3), $7.35(1 \mathrm{H}, \mathrm{t}$, $J \cong 7.5 \mathrm{~Hz}, \mathrm{H}-6), 7.53(1 \mathrm{H}, \mathrm{t}, J \cong 7.5 \mathrm{~Hz}, \mathrm{H}-7), 7.83(1 \mathrm{H}, \mathrm{d}, J \cong 9.0 \mathrm{~Hz}, \mathrm{H}-5), 7.75(1 \mathrm{H}, \mathrm{d}, J \cong 8.0 \mathrm{~Hz}$,

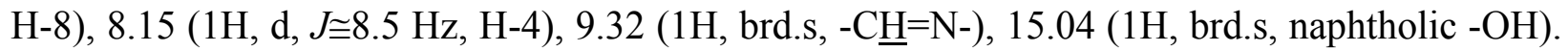
${ }^{13} \mathrm{C}$ NMR (400 MHz, CDCl $): \delta 21.2\left(-\mathrm{CH}_{3}\right), 91.3\left(\mathrm{C}-6^{\prime}\right), 109.2(\mathrm{C}-1), 119.2(\mathrm{C}-3), 119.3\left(\mathrm{C}-2^{\prime}\right)$, 120.7 (C-8), 123.7 (C-6), 127.5 (C-4a), 128.9 (C-4'), 129.4 (C-5), 133.0 (C-8a), 136.0 (C-4), $139.3\left(\mathrm{C}-5^{\prime}\right), 139.8\left(\mathrm{C}-3^{\prime}\right), 148.7\left(\mathrm{C}-1^{\prime}\right), 157.3(-\mathrm{CH}=\mathrm{N}-), 165.9(\mathrm{C}-2)$.

1-\{[(2-iodo-4-methylphenyl)imino]methyl\}-2-naphthol (2e). Yield: $41.8 \%$. mp: $142{ }^{\circ} \mathrm{C}$. Mass spectra: $\mathrm{m} / \mathrm{z}=\mathrm{M}^{+}$[387.0], [259.0 (M-HI, 36.1\%] $]^{+},\left[106.1\left(\mathrm{C}_{8} \mathrm{H}_{10}, 100 \%\right)\right]^{+}$. Anal: Calcd for $\mathrm{C}_{18} \mathrm{H}_{14} \mathrm{INO}$ (387.21): C, 55.83; H, 3.64; N, 3.62. Found: C, 54.10; H, 3.69; N, 3.41. IR (KBr) $v_{\max }: 3438,1625,1574-1497 \mathrm{~cm}^{-1}$. UV (EtOH) $\lambda_{\max }(\log \varepsilon): 287.6(0.970), 312.8(0.779), 366.4$ (0.562), 442.4 (0.207), $463.6(0.196) \mathrm{nm} .{ }^{1} \mathrm{H}-\mathrm{NMR}\left(400 \mathrm{MHz}, \mathrm{CDCl}_{3}\right): \delta 2.36\left(3 \mathrm{H}, \mathrm{s},-\mathrm{CH}_{3}\right)$, $7.15\left(1 \mathrm{H}, \mathrm{d}, J=7.5 \mathrm{~Hz}, \mathrm{H}-2^{\prime}\right), 7.20\left(1 \mathrm{H}, \mathrm{d}, J=9.0 \mathrm{~Hz}, \mathrm{H}-5^{\prime}\right), 7.24(1 \mathrm{H}, \mathrm{d}, J=7.5 \mathrm{~Hz}, \mathrm{H}-3), 7.32$ $(1 \mathrm{H}, \mathrm{t}, J=7.5 \mathrm{~Hz}, \mathrm{H}-6), 7.50(1 \mathrm{H}, \mathrm{t}, J \cong 7.5 \mathrm{~Hz}, \mathrm{H}-7), 7.73\left(1 \mathrm{H}, \mathrm{s}, \mathrm{H}-3^{\prime}\right), 7.75(1 \mathrm{H}, \mathrm{d}, J=8.5 \mathrm{~Hz}, \mathrm{H}-$ 8), $7.79(1 \mathrm{H}, \mathrm{d}, J=9.0 \mathrm{~Hz}, \mathrm{H}-5), 8.10(1 \mathrm{H}, \mathrm{d}, J=8.5, \mathrm{H}-4), 9.29(1 \mathrm{H}$, brd.s, $-\mathrm{C} \underline{\mathrm{H}}=\mathrm{N}) 15.06(1 \mathrm{H}$, brd.s, naphtholic -OH). ${ }^{13} \mathrm{C}$ NMR $\left(400 \mathrm{MHz}, \mathrm{CDCl}_{3}\right): \delta 20.4\left(-\mathrm{CH}_{3}\right), 95.6\left(\mathrm{C}-6{ }^{\prime}\right), 109.2(\mathrm{C}-1)$, 117.9 (C-2'), 119.1 (C-3), 120.6 (C-8), 123.6 (C-6), 127.7 (C-4a), 127.9 (C-7), 129.3 (C-5), 130.2 (C-5'), 132.9 (C-8a), 135.8 (C-4), $138.2\left(\mathrm{C}-4^{\prime}\right), 139.9\left(\mathrm{C}-3^{\prime}\right), 146.4\left(\mathrm{C}-1^{\prime}\right), 156.8(-\mathrm{CH}=\mathrm{N}-$ ), 165.5 (C-2). HETCOR $\left(\mathrm{CDCl}_{3}\right): 9.29,-\mathrm{CH}=\mathrm{N}-(156.8,-\mathrm{CH}=\mathrm{N}$ - azomethine carbon), 8.10, H-4 (135.8, C-4), 7.75, H-8 (120.6, C-8), 7.73, H-3' (139.9, C-3'), 7.79, H-5 (129.3, C-5), 7.50, H-7 (127.9, C-7), 7.32, H-6 (123.6, C-6), 7.20, H-5' (130.2, C-5'), 7.24, H-3 (119.1, C-3), 7.15, H-2' $\left(117.9, \mathrm{C}-2^{\prime}\right)$.

\section{Acknowledgements}

This work was supported by the Eskisehir Osmangazi University Scientific Research Project Commission (Grant No. 200419036).

\section{References}

1. Merkushev, E. B. Synthesis 1988, 923.

2. Olah, G. A.; Wang, Q.; Prakash, G. K. J.Org. Chem. 1993, 58, 3194. 
3. Wirth, H. O.; Konigslen, O.; Kern, W. Liebigs Ann. Chem. 1960, 634, 84.

4. Zweig, A.; Huffman, K. R.; Nachtigall, G. W. J. Org. Chem. 1977, 42, 4049.

5. Suzuki, H.; Haruta, Y. Bull. Chem. Soc. Jpn. 1973, 46, 589.

6. Pizey, J. S. In Synthetic Reagents; Wiley: New York, 1977; Vol. 3, p 227.

7. Sugita, T.; Idei, M.; Takegami, Y. Chem. Lett. 1982, 1481.

8. Boothe, R.; Dial, C.; Conaway, R.; Pagni, R. M.; Kabalka, G. W. Tetrahedron Lett. 1986, 27, 2207.

9. Suzuki, H. Org. Synt. 1988, 4, 700.

10. Elbs, K.; Jaroslawzew, A. J. Prakt. Chem. 1913, 88, 92.

11. Marko, D. M.; Belyaev, Y. A. Khim. Referat. Zhur. 1941, 4, 49.

12. Edgar, K. J.; Falling, S. N. J. Org. Chem. 1990, 55, 5287.

13. Barluenga, J.; Gonzalez, J. M.; Garcia-Martin, M. A.; Campos, P. J.; Asensio, G. J. Org. Chem. 1993, 58, 2058.

14. Sy, W. W. Tetrahedron Lett. 1993, 34, 6223.

15. Bachki, A.; Foubelo, F.; Yus, M. Tetrahedron 1994, 50, 5139.

16. Hubig, S. M.; Jung, W.; Kochi, J. K. J. Org. Chem. 1994, 59, 6233.

17. Orito, K.; Hatakeyama, T.; Takeo, M.; Suginome, H. Synthesis 1995, 1273.

18. Brunel, Y.; Rousseau, G. Tetrahedron Lett. 1995, 45, 8217.

19. Brazdil, I. C.; Cutier, C. J. J. Org. Chem. 1996, 61, 9621.

20. Carreno, M. C.; Ruano, J. L. G.; Sanz, G.; Toledo, M. A.; Urbano, A. Tetrahedron Lett. 1996, 37, 4081.

21. Noda, Y.; Kashima, M. Tetrahedron Lett. 1997, 38, 6225.

22. Chiappe, C.; Pieraccini, D. ARKIVOC 2002, (xi), 249.

23. Patil, B. R.; Bhusare, R. P; Vibhute, Y. B. ARKIVOC 2006, (i), 104.

24. Blackmore, I. J.; Boa, A. N.; Murray, E. J.; Dennis, M.; Woodward, S. Tetrahedron Lett. 1999, 40, 6671 .

25. Krishna Mohan, K. V. V.; Narender, N.; Kulkarni, S. J. Tetrahedron Lett. 2004, 45, 8015.

26. Johnson, R.; Meijer, A.; Ellervik, U. Tetrahedron 2005, 61, 11656.

27. Sayyed, M. A.; Mokle, S. S.; Vibhute, Y. B. ARKIVOC 2006, (xi), 221.

28. Castanet, A. S.; Colobert, F.; Broutio, P. E. Tetrahedron Lett. 2002, 43, 5047.

29. Firouzabadi, H.; Iranpoor, N.; Shiri, M. Tetrahedron Lett. 2003, 44, 8781.

30. Kabalka, G. W.; Mereddy, A. R. Tetrahedron Lett. 2004, 45, 343.

31. Lokhande, P. D. and Ghiya, B. J. Indian Chem. Soc. 1991, 68,412.

32. Sawich, A., Zetensow, J. V. Astron Fiz. Khim. Spit Syn. I 1956, 1, 233. 